\title{
ORTHODONTIC MANAGEMENT OF A CROWDED CLASS III MALOCCLUSION ON A CLASS III SKELETAL BASE: A CASE REPORT
}

W.N. Wan Hassan. Orthodontic management of a crowded Class III malocclusion on a Class III skeletal base: A case report. Annal Dent Univ Malaya 2010; 17: $40-49$.

\begin{abstract}
A late adolescent patient presented with a Class III malocclusion on a skeletal Class III base, complicated by severe upper arch and moderate lower arch crowding, reverse overjet, anterior and bilateral posterior crossbites with displacement, proclined upper incisors, retroclined lower incisors, distally tipped lower canines and non-coincident centrelines. Treatment was undertaken on an extraction basis by employing the use of an upper removable appliance with Z-springs and posterior bite blocks to correct the anterior crossbite, quad helix and jockey arch for arch expansion, and pre-adjusted edgewise fixed appliance to level and align, space closure and achieve a mutually protective functional occlusion. This paper discussed the rational and evidences behind the treatment employed.
\end{abstract}

Key words: Class III, orthodontic camouflage

\section{INTRODUCTION}

In Class III malocclusion, the lower incisor edge lies anterior to the cingulum plateau of the palatal surface of the upper incisors (1). Class III malocclusion often presents with a Class III skeletal base relationship. The Class III relationship is thought to be due to polygenic multifactorial inheritance with variable mode of transmissions although there has been suggestions that environmental factors such as enlarged tonsils and nasal blockages may contribute to mandibular prognatism (2). Generally the soft tissues are regarded to be of lesser importance in the aetiology of the malocclusion. The lip and tongue pressures are thought to influence the dental inclinations to compensate for the underlying skeletal discrepancy.

Early treatment of the skeletal and dental Class III relationships could be addressed orthopaedically such as by the use of facemask with rapid palatal expansion $(3,4)$ which has been shown to demonstrate long term favourable improvement in the skeletal relationship (5). In older patients with moderate to severe skeletal Class III pattern, cases usually do not camouflage well to conceal the skeletal problem and may need combined orthodontic-orthognathic treatment.

When making a decision to treat by orthodontics alone, Proffit and colleagues (2007) suggested that the
Case Report

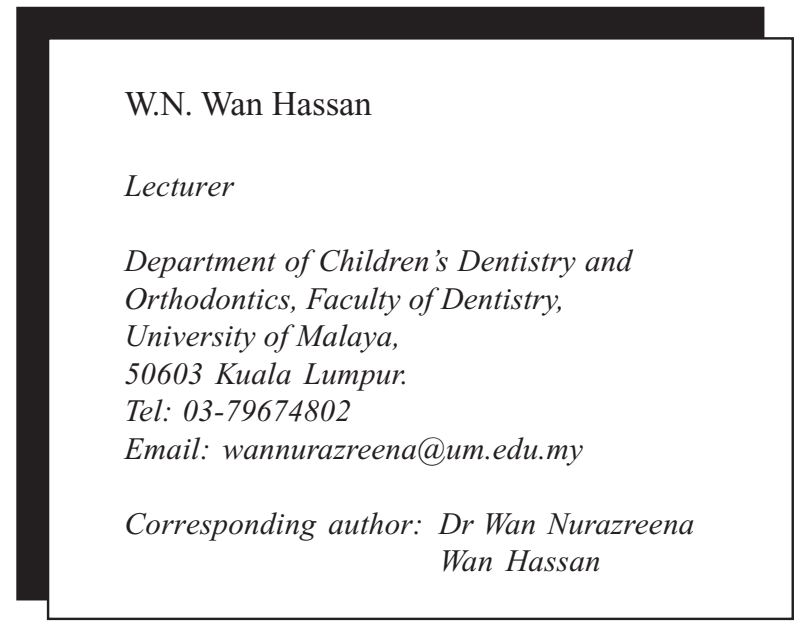

good characteristics for camouflage treatment were mild or mild to moderate skeletal Class III patients who have passed their peak pubertal growth spurt with good vertical proportions and reasonably good alignment of the teeth. It was also outlined that camouflage treatment should be avoided in cases with severe or moderate to severe skeletal Class III, vertical skeletal discrepancies, severe crowding or protrusion of the incisors, in adolescents with potential growth and in non-growing adults with more than mild discrepancies where surgery may offer better long-term results (6). Cephalometrically, the suggested thresholds below which surgery was decided include ANB value of $-4^{\circ}$, lower incisor angulation of $83^{\circ}$, maxillary to mandibular ratio of 0.84 and Holdaway angle of $3.5^{\circ}$ (7).

These were good guidelines for treatment planning but the decision should be made on individual basis. This paper will present a case of a late adolescent patient who presented with Class III malocclusion on a skeletal Class III pattern, complicated by crowding and existing dentoalveolar compensation that was borderline for orthodontic-orthognathic treatment but was treated by orthodontic camouflage.

\section{CASE REPORT}

A 16 years and 3 months old male attended the orthodontic clinic and complained that his front teeth were not straight. On clinical examination he presented with a Class III malocclusion on a mild skeletal Class III base with average lower face height and Frankfort mandibular plane angle. There was no significant transverse discrepancy. The lips were competent at rest with a low smile line and the nasiolabial angle was obtuse. 
The oral hygiene was fair with BPE scores of 0 in all sextants apart from the lower labial segment which scored as 2 due to the presence of calculus. All permanent teeth were present apart from the third molars in all quadrants (Figure 1). The lower arch was moderately crowded with retroclined lower incisors and distally inclined lower canines. The upper arch was severely crowded with a palatally erupted $\underline{\underline{2}}$ and proclined upper incisors. In occlusion, the reverse overjet was $2 \mathrm{~mm}$ and the overbite was average. The molar relationships were $1 / 4$ units Class III on the left side and Class I on the right side. The canine relationships were Class I on the left side and $1 / 4$ units Class II on the right side. The centreline was not coincident with the upper centreline shifted to the left by $2 \mathrm{~mm}$ relative to the facial midline. Teeth in crossbites were:

\begin{tabular}{|c|c|c|c|c|c|c|c|c|c|}
\hline 7 & 6 & 5 & & 2 & 1 & 1 & & 5 & 6 \\
\hline 7 & 6 & 5 & 3 & 2 & 1 & 1 & 3 & & 6 \\
\hline
\end{tabular}

On centric relation, there was an initial contact between the upper left lateral and lower left central incisor. The mandible then displaced anteriorly by $2 \mathrm{~mm}$ into maximum intercuspation.

The dental panoramic tomogram showed normal dental development and presence of all third permanent molars (Figure 2). The lateral cephalometric analysis (Table 1) confirmed the Class III skeletal relationship. Cervical spine maturation stage was at cervical stage 5 (CS 5). This was evident by the concavity at the lower borders of the $\mathrm{C} 2, \mathrm{C} 3$ and $\mathrm{C} 4$, and both $\mathrm{C} 3$ and $\mathrm{C} 4$ being squared in shape (8) (Figure $3)$. This suggested that the peak mandibular growth has ended at least 1 year before this stage.

Family history indicated that his father had a mild Class III skeletal pattern. However, none of his immediate family had undergone orthodontic or orthognathic treatment before.

Treatment aims included to ensure optimum oral hygiene and dental health and to monitor his growth

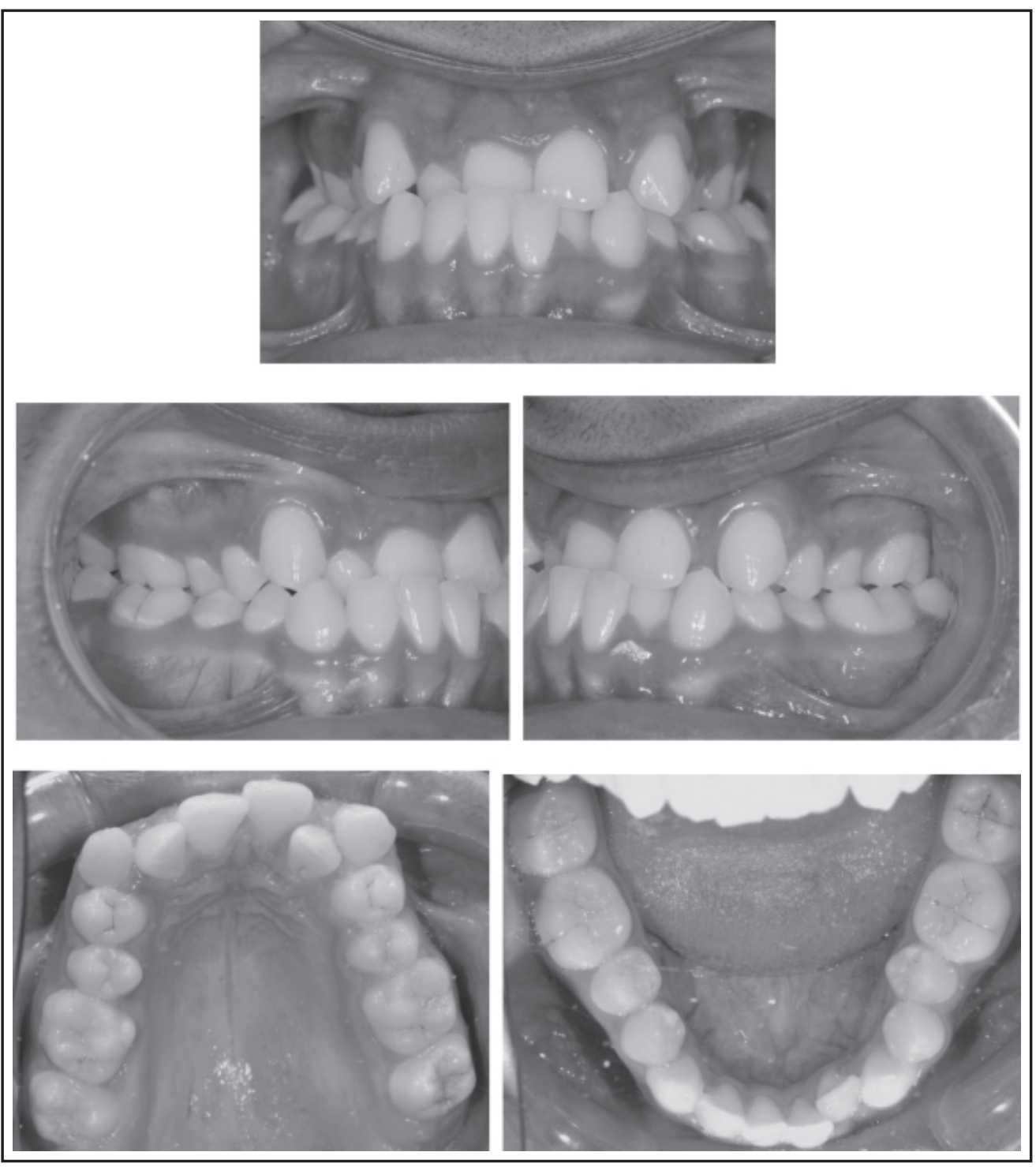

Figure 1. Pre-treatment intra-oral views in centric occlusion (From the top clockwise: anterior view, left buccal view, mandibular occlusal view, maxillary occlusal view and right buccal view). 


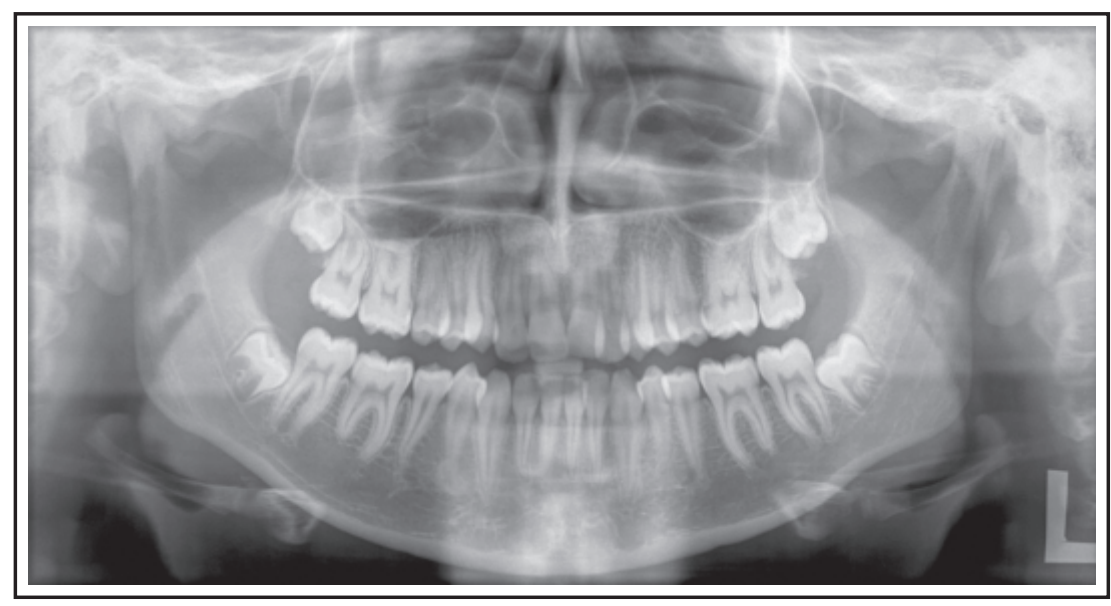

Figure 2. Dental panoramic tomogram showing normal dental development and presence of all third permanent molars.

Table 1. Pre-treatment cephalometric values

\begin{tabular}{lcc}
\hline VARIABLE & PRE-TREATMENT & NORMAL \\
\hline SNA & $81.2^{\circ}$ & $82^{\circ} \pm 3$ \\
SNB & $86.2^{\circ}$ & $79^{\circ} \pm 3$ \\
ANB & $-5.0^{\circ}$ & $3^{\circ} \pm 1$ \\
Wits appraisal & $-7.5 \mathrm{~mm}$ & $-1 \mathrm{~mm}$ \\
Upper incisor to maxillary plane angle & $119.5^{\circ}$ & $108^{\circ} \pm 5$ \\
Lower incisor to mandibular plane angle & $82.4^{\circ}$ & $92^{\circ} \pm 5$ \\
Interincisal angle & $137.6^{\circ}$ & $133^{\circ} \pm 10$ \\
Maxillary mandibular planes angle & $20.5^{\circ}$ & $27^{\circ} \pm 5$ \\
Upper anterior face height & $47 \mathrm{~mm}$ & $55 \mathrm{~mm} \pm 3$ \\
Lower anterior face height & $60 \mathrm{~mm}$ & $70.5 \mathrm{~mm} \pm 4.5$ \\
Face height ratio & $56 \%$ & $55^{\circ} \%$ \\
Lower incisor to APo line & $3.4 \mathrm{~mm}$ & $0-2 \mathrm{~mm}$ \\
Lower lip to Ricketts E Plane & $-5.3 \mathrm{~mm}$ & $0 \mathrm{~mm} \pm 3^{\mathrm{a}}$ \\
\hline
\end{tabular}

Sources of normal values: Jacobson (1975) Am J Orthod. 67:125-133.

Houston WJB, Stephens CD \& Tulley WJ (1992) A textbook of orthodontics. Wright, Oxford

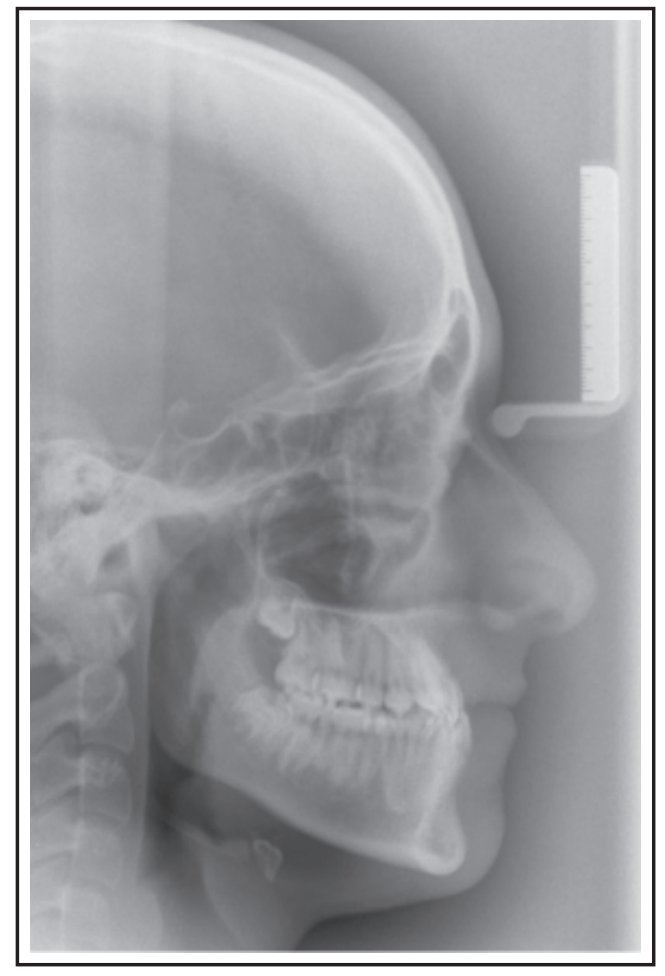

Figure 3. Lateral cephalometric radiograph showing the cervical spine maturation stage at CS5. throughout treatment. Treatment aimed to address the tooth arch length discrepancy, level and align both arches, correct the inter arch relationships achieving a mutually protective functional occlusion and to provide appropriate retention after fixed appliances.

Treatment by orthodontic camouflage was decided and the treatment plan included:

1. Oral hygiene instruction

2. Growth monitoring by the use of height and weight charts.

3. Upper removable appliance (URA) with posterior blocks to disclude the occlusion and Z-springs to procline the $21 / 1$

4. Quad helix

5. Reassessment

After 4 months of treatment with the URA, positive overjet of the $21 / 1$ and overbite were achieved. This was followed by the use of quad helix to expand the upper arch. Nine months into treatment, his height had not changed suggesting no further growth has occurred. Lateral cephalometric assessment showed that the upper incisors had further proclined to $128.2^{\circ}$ (Table 2). In view of the severity of the crowding and the incisor inclination, stage 2 treatment plan included: 
Table 2. Mid-treatment cephalometric values

\begin{tabular}{lcc}
\hline VARIABLE & MID-TREATMENT & CHANGE \\
\hline SNA & $80.5^{\circ}$ & $-0.7^{\circ}$ \\
SNB & $85.0^{\circ}$ & $-1.2^{\circ}$ \\
ANB & $-4.6^{\circ}$ & $+0.4^{\circ}$ \\
Wits appraisal & $-5.3 \mathrm{~mm}$ & $+2.2 \mathrm{~mm}$ \\
Upper incisor to maxillary plane angle & $128.2^{\circ}$ & $+8.7^{\circ}$ \\
Lower incisor to mandibular plane angle & $81.1^{\circ}$ & $-1.3^{\circ}$ \\
Interincisal angle & $131.2^{\circ}$ & $-6.4^{\circ}$ \\
Maxillary mandibular plane angle & $19.5^{\circ}$ & $-1^{\circ}$ \\
Upper anterior face height & $47.3 \mathrm{~mm}$ & $+0.3 \mathrm{~mm}$ \\
Lower anterior face height & $60.2 \mathrm{~mm}$ & $+0.2 \mathrm{~mm}$ \\
Face height ratio & $56 \%$ & $0 \%$ \\
Lower incisor to APo line & $1.9 \mathrm{~mm}$ & $-1.5 \mathrm{~mm}$ \\
Lower lip to Ricketts E Plane & $-2.4 \mathrm{~mm}$ & $+2.9 \mathrm{~mm}$ \\
\hline
\end{tabular}

1. Extractions of all first permanent premolars

2. Upper and lower pre-adjusted edgewise fixed appliance $\left(\mathrm{MBT}^{\mathrm{TM}}\right.$ prescription, 0.022 " $\mathrm{x}$ 0.028 " slot)

3. Retention with removable Hawley retainers and a lower bonded retainer.

$\mathrm{MBT}^{\mathrm{TM}}$ Versatile+ (3M Unitek) brackets and bands were fitted in the upper and lower arches following extraction. The $7 \backslash 27$ were initially excluded. The 0.012 " nickel titanium archwire was fitted with lacebacks on all quadrants during the initial levelling and alignment stages (Figure 4). A progression of light nickel titanium archwires were used to reach 0.017 ' $\mathrm{x}$ 0.025 " stainless steel archwire in the upper arch and 0.020 " stainless steel archwire with loops in the lower arch. Nickel titanium push coil spring was used to open the space for the $\backslash \underline{2}$. Once there was sufficient space, the 12 bracket was inverted and bonded. Light nickel titanium archwires (0.012") were used to a progression of up to 0.019 " $\mathrm{x} 0.025$ " posted stainless steel archwire. Glass ionomer cement was placed on the lower first permanent molars to disclude the occlusion during alignment of the $\underline{2}$, which was later removed once the tooth was in a positive overjet and overbite relationship.

At 16 months into treatment, the quad helix was removed and space closure commenced using elastomeric power chains. When the spaces had closed, there was still displacement due to the crossbites on the second permanent molars. The $\underline{7 \backslash 7}$ brackets were bonded. A jockey arch $(0.8 \mathrm{~mm}$ stainless steel) was

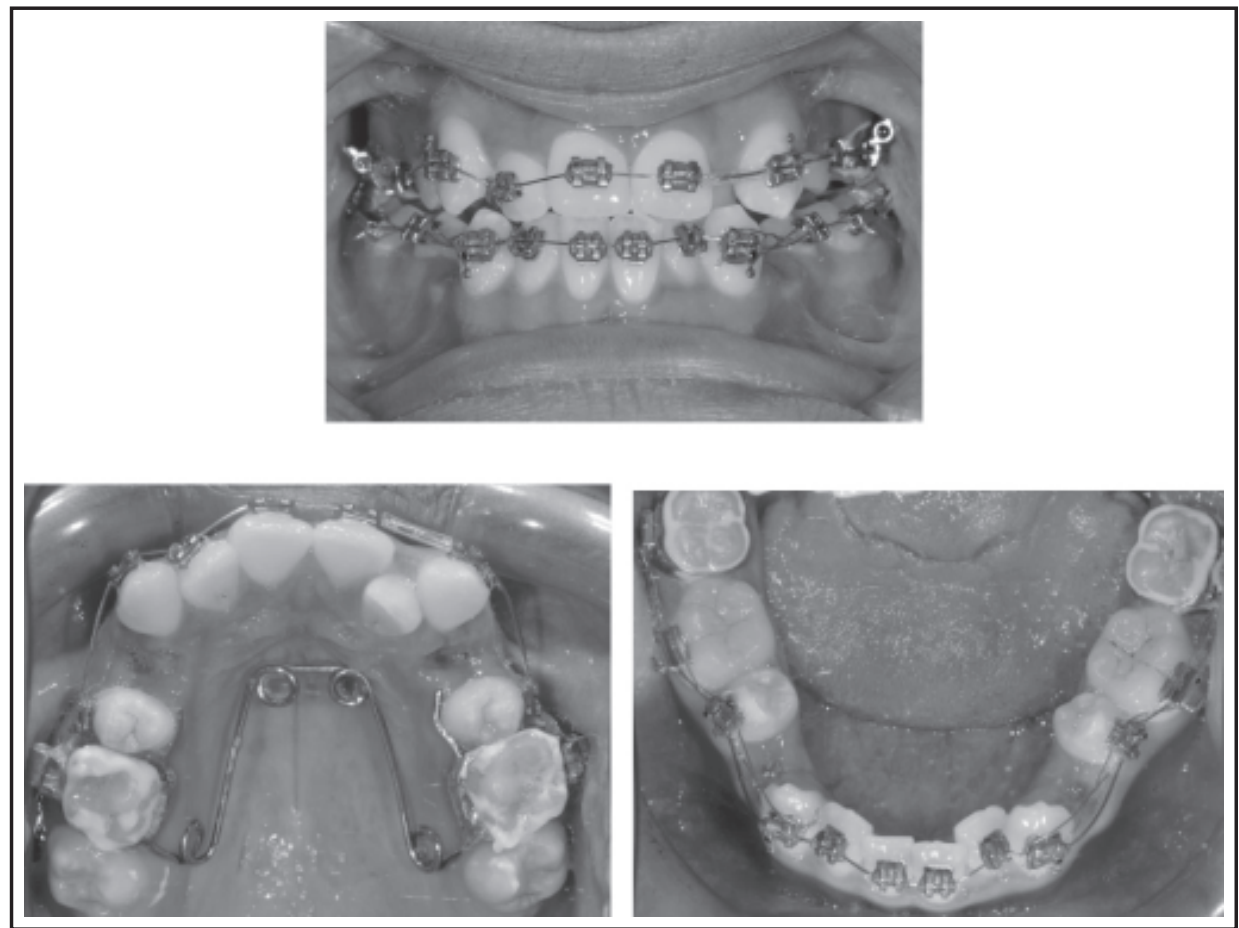

Figure 4. Intraoral views showing upper and lower pre-adjusted edgewise appliance with 0.012 " nickel titanium archwires for initial alignment. Quad helix was previously fitted for arch expansion (From the top clockwise: anterior viewmandibular occlusal view and maxillary occlusal view). 
inserted into the extra-oral traction tubes and secured with separators to support and aid further expansion of the upper arch (Figure 5). The teeth were aligned using 0.012 " nickel titanium archwire followed by upper 0.016 " stainless steel archwire with passive intrusion bends to prevent extrusion of the $\underline{7 \backslash 7}$. Centre-line and buccal segment corrections were done by judicious use of intermaxillary Classs III elastics.

Near end of treatment lateral cephalometric radiograph was taken to assess the incisor inclinations (Table 3). Cervical spine maturation assessment indicated cervical stage 6 (CS 6), which was evident by the concavity at the lower borders of the $\mathrm{C} 2, \mathrm{C} 3$ and $\mathrm{C} 4$, and both $\mathrm{C} 3$ and $\mathrm{C} 4$ being rectangular vertical in shape (Figure 6) and suggested that the peak mandibular growth has ended at least 2 year before this stage (8). After 25 months of treatment, the fixed appliances were removed (Figure 7). The patient was provided with bonded lower retainer. Upper and lower Hawley retainers were also fitted to be worn for 6 months full time and a further 6 months of night time wear.

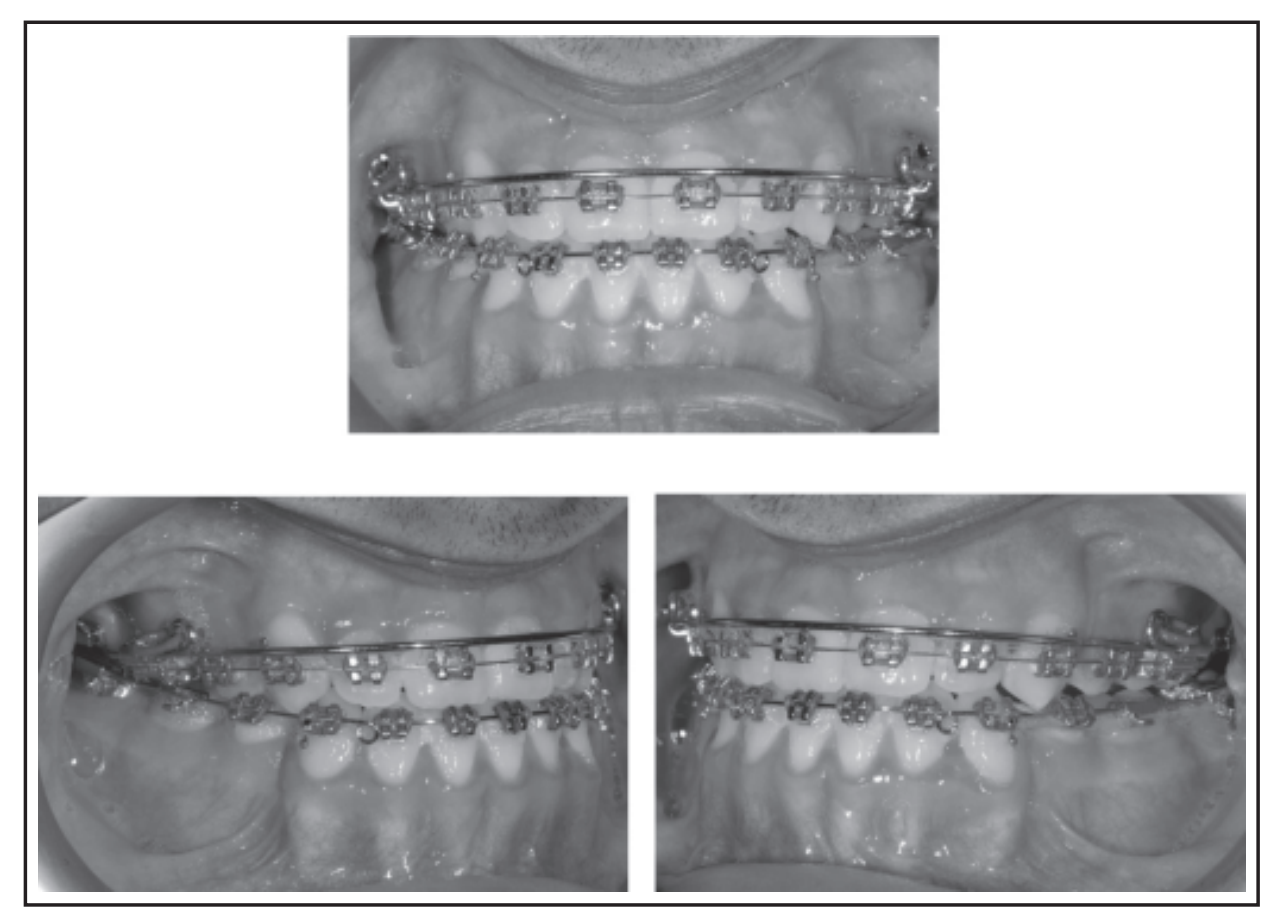

Figure 5. Jockey arch used to support and facilitate concurrent expansion of the upper arch during alignment of the $\underline{7 \backslash 7}$ (From the top clockwise: anterior view, left buccal view and right buccal view).

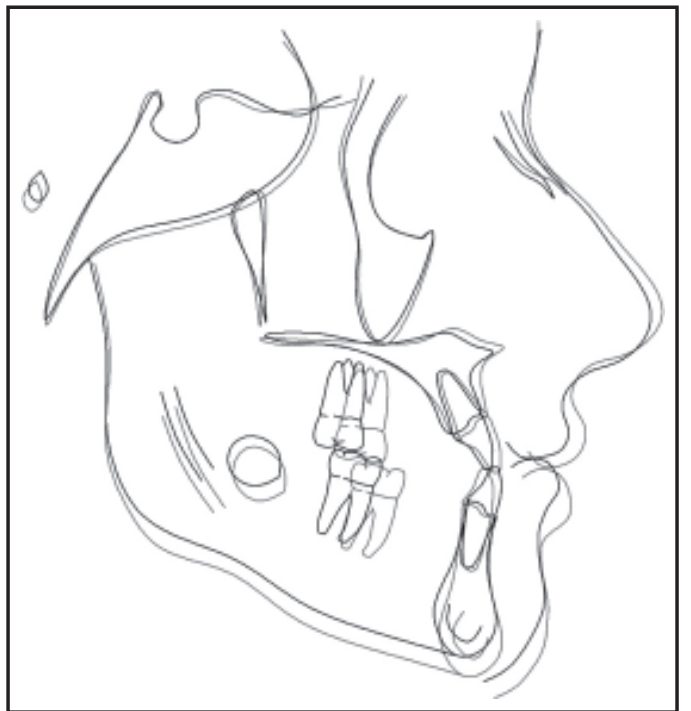

Figure 5. Overall superimposition of the pre-treatment and near end of treatment lateral cephalometrics registered at on the Sella-Nasion line at Sella.

(Pre-treatment _ - Near end of treatment
Table 3. Near end of treatment cephalometric values

\begin{tabular}{lcc}
\hline VARIABLE & $\begin{array}{c}\text { NEAR END OF } \\
\text { TREATMENT }\end{array}$ & CHANGE \\
\hline SNA & $81.9^{\circ}$ & $+0.7^{\circ}$ \\
SNB & $85.6^{\circ}$ & $-0.6^{\circ}$ \\
ANB & $-3.7^{\circ}$ & $+1.3^{\circ}$ \\
Wits appraisal & $-3.6 \mathrm{~mm}$ & $+3.9 \mathrm{~mm}$ \\
Upper incisor to maxillary & $119.2^{\circ}$ & $-0.3^{\circ}$ \\
plane angle & & \\
Lower incisor to mandibular & $75.2^{\circ}$ & $-7.2^{\circ}$ \\
plane angle & & \\
Interincisal angle & $144.3^{\circ}$ & $+6.7^{\circ}$ \\
Maxillary mandibular & $21.4^{\circ}$ & $+0.9^{\circ}$ \\
plane angle & & \\
Upper anterior face height & $46.2 \mathrm{~mm}$ & $-0.8 \mathrm{~mm}$ \\
Lower anterior face height & $64.7 \mathrm{~mm}$ & $+4.7 \mathrm{~mm}$ \\
Face height ratio & $58.4 \%$ & $+2.4 \%$ \\
Lower incisor to APo line & $-0.6 \mathrm{~mm}$ & $-4 \mathrm{~mm}$ \\
Lower lip to Ricketts E Plane & $-3.4 \mathrm{~mm}$ & $+1.9 \mathrm{~mm}$ \\
\hline
\end{tabular}




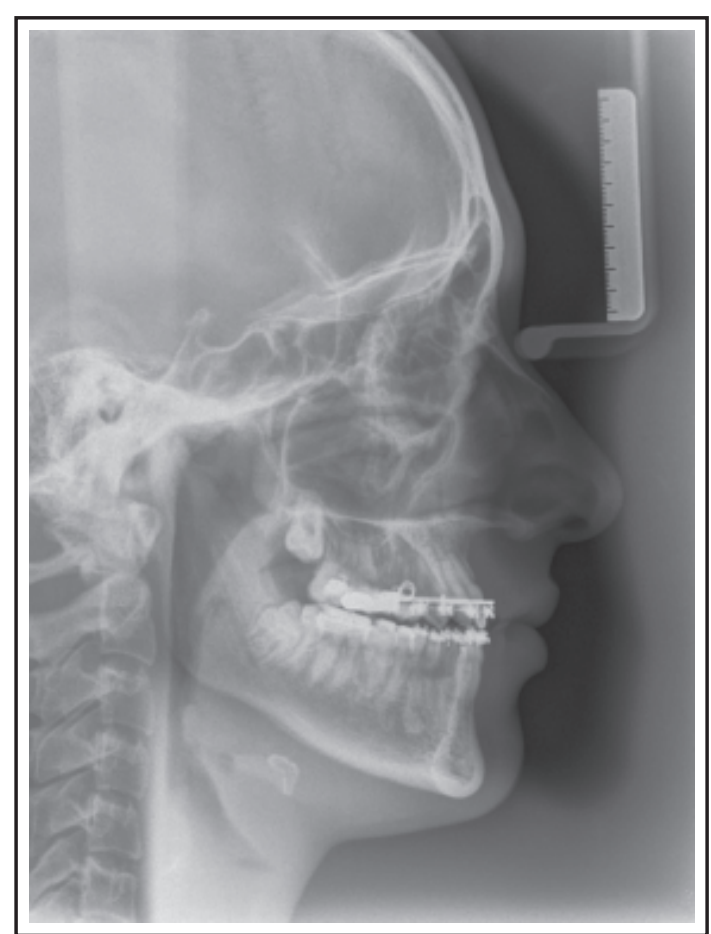

Figure 6. Lateral cephalometric radiograph showing the cervical spine maturation stage at CS6.

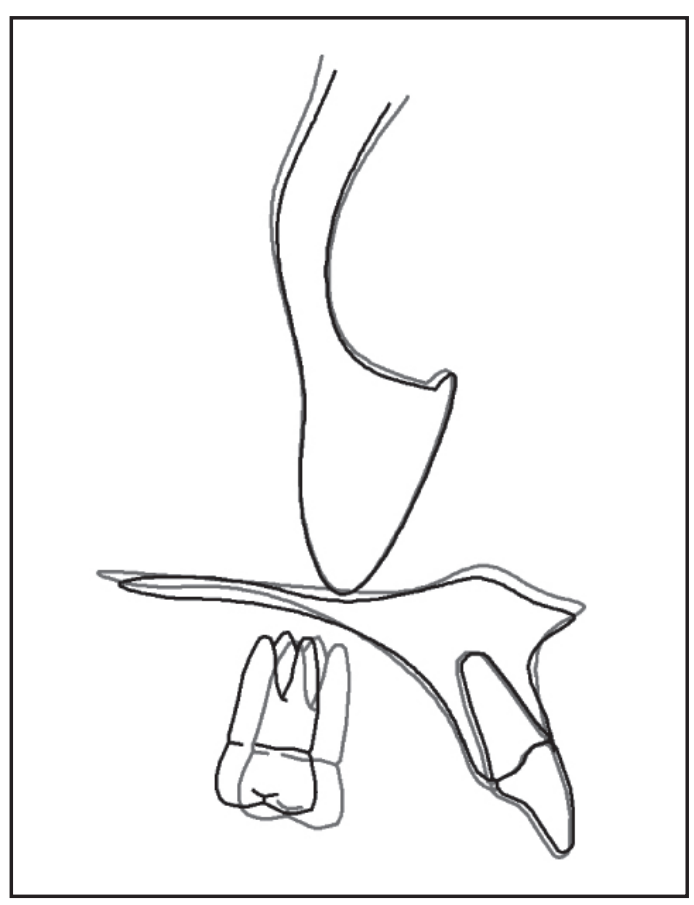

Figure 6. Cephalometric superimposition of the maxilla registered at the maxillary key ridge.

(Pre-treatment — ; Near end of treatment

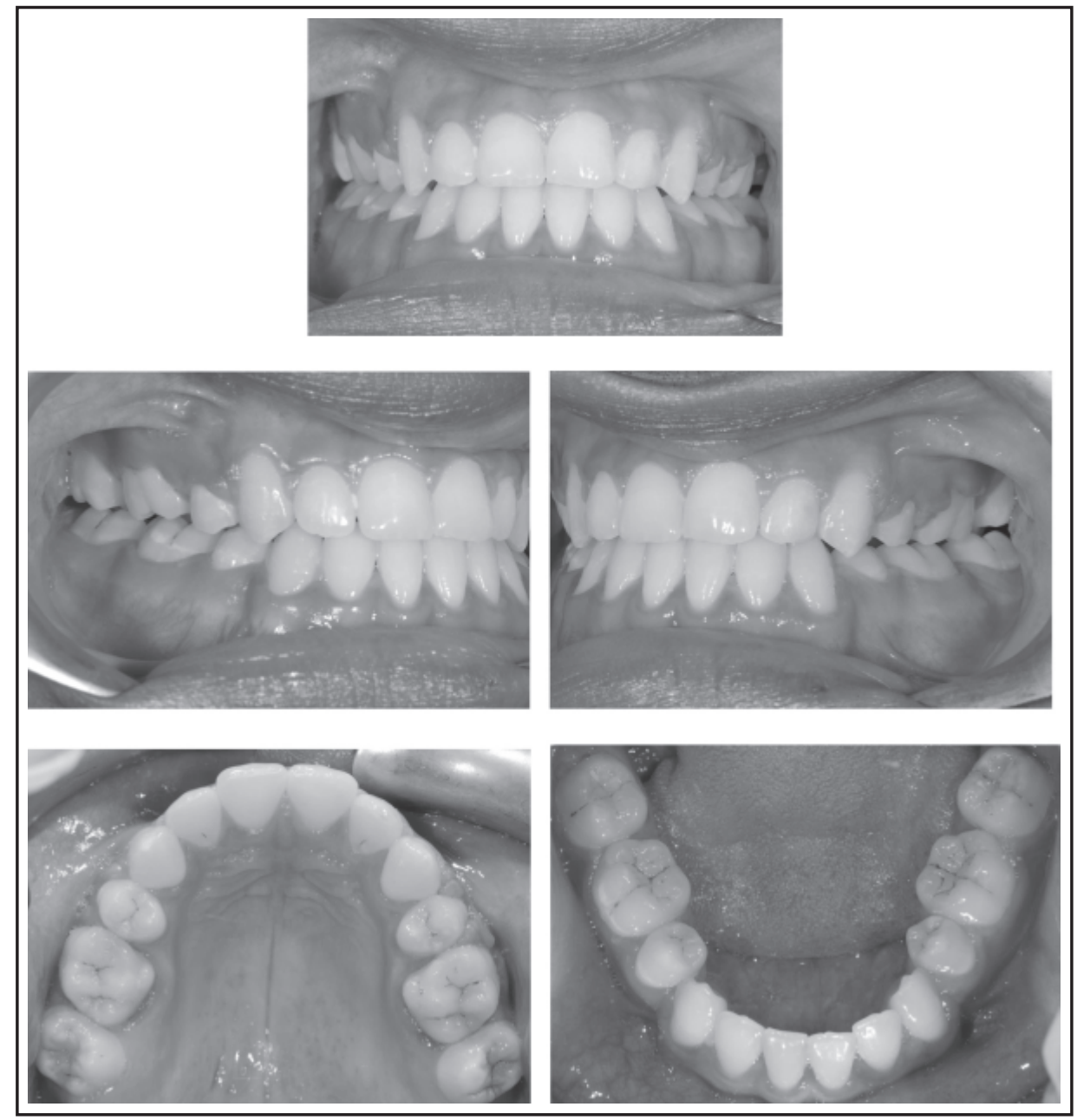

Figure 7. Post treatment intraoral views (From the top clockwise: anterior view, left buccal view, mandibular occlusal view, maxillary occlusal view and right buccal view). 


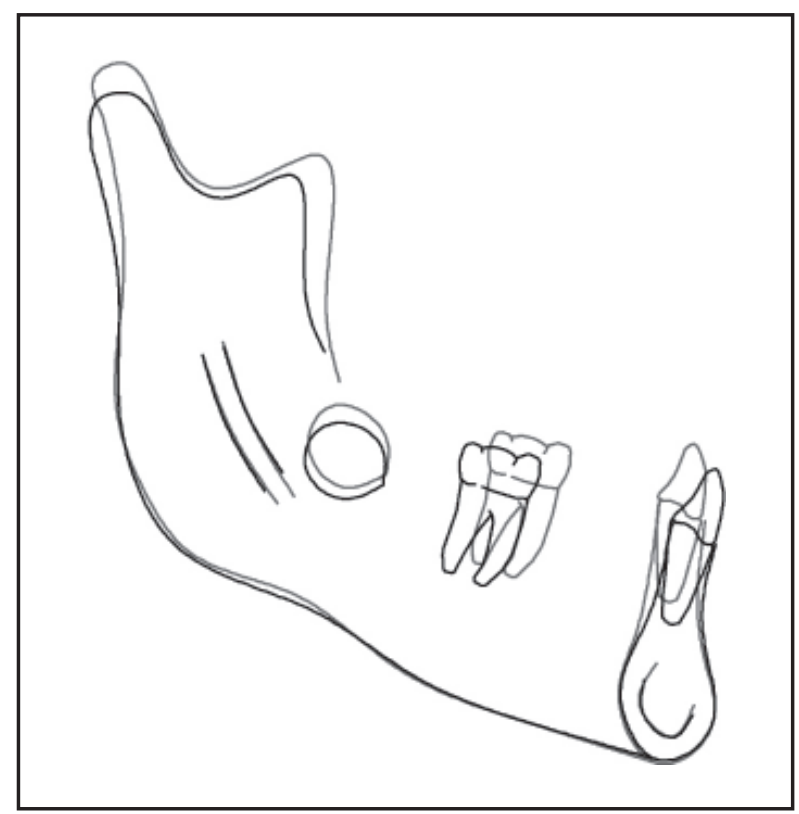

Figure 7. Mandibular superimposition registered at Bjork's structures of the mandible on the inferior dental canal and inner cortical outline of the mandibular symphysis.

(Pre-treatment — ; Near end of treatment ——)

\section{DISCUSSION}

The patient presented in the late adolescent age of 16 years and 3 months with a cervical spine maturation assessment at stage CS5, which suggested that his pubertal growth spurt has passed by at least 1 year. The severity of the malocclusion and existing dentoalveolar compensation to camouflage the underlying skeletal discrepancy suggested borderline orthodontic camouflage or orthognathic treatment approach. This was supported by the cephalometric finding of an ANB value of $-5^{\circ}$ and lower incisor inclination of $82^{\circ}$ which were just below the suggested threshold for treatment by surgical approach (7). The extra-oral clinical examination was mild skeletal Class III pattern with an aesthetically acceptable facial profile and was much more favourable than that implied from the cephalometric assessment.

When making a decision for treatment, it is important to obtain informed consent. For consent to be valid, the patient should be explained of the proposed treatment, the risks involved and the alternative treatments (9). The parent and/or legal guardian should be included if the patient is below the appropriate legal age. Class III cases, particularly those with moderate or severe skeletal dysplasia, usually do not camouflage well (6). On the other hand, orthognathic treatment has the risks of increased morbidity associated with surgical treatments such as loss of sensation (temporary or permanent parasthesia or anaesthesia) of the nerves where the surgical cut was made and risks associated with the general anaesthesia in general. Stability of treatment was also dependent on the type of surgical movement involved. Surgery to move the maxilla forward was found to be stable first year post surgery while concurrent forward movement of the maxilla and backward movement of the mandible was found to be stable only with rigid fixation, and single jaw movement to bring the mandible back was found to be less stable (10). The treatments options and risks were discussed with the patient, who then declined to undergo orthognathic surgery and opted treatment to be done by orthodontics alone.

Quite often a dilemma may arise in deciding the most suitable time to treat adolescents with skeletal Class III pattern who has passed their peak pubertal growth. Unlike patients with skeletal Class II pattern, any late mandibular growth may be unfavourable to the treatment. Baccetti and colleagues (2007) found that the total mandibular length increased 3 times in boys compared with those with normal occlusion between CS4 to CS6. However, the significance of this growth to affect the anterio-posterior relationship (e.g. ANB) was not discussed. On hindsight, perhaps it may have been prudent to wait for a couple more years to monitor for any potential late mandibular growth. Nevertheless, the patient's near end of treatment cephalogram showed little change in the ANB value. The Wits appraisal was increased by $3.9 \mathrm{~mm}$, which suggested a reduced skeletal Class III relationship. The changes were probably due to elimination of the anterior displacement which has resulted in the mandible to be recorded in a more retruded position relative to the initial lateral cephalogram and/or changes due to dentoalveolar remodelling of the $\mathrm{B}$ point following lower incisor retroclination. The overall superimposition (Figure 5) showed little significant antero-posterior change, suggesting that postponing the treatment to monitor for the late mandibular growth would have been of little difference in this case. Vertically, there was a small change in the lower anterior face height. This may have been a reflection of vertical growth and/or the effect of the inter-arch mechanics used which had extruded the lower molars and incisors, thus displacing the mandible downwards. Growth monitoring by the use of height chart showed that there was no change in the patient's height throughout treatment until the most recent record at debond. The lack of continued somatic growth in the past 2 years and sagittal mandibular growth, supported by the cervical spine maturation stage 6 of the near end of treatment cephalogram suggested that his growth has slowed down. Therefore, the prognosis for stability was deemed to be favourable.

It was important to reinforce strict oral hygiene measures prior to and during treatment as orthodontic treatment could increase the risk of enamel demineralisation and white spot lesions and sometimes remain after treatment (11). Prior to treatment, the patient already had white spot lesions at the cervical margins of the lower right first 
permanent molar, second and first premolars and the lower left second permanent premolar, suggesting that he had a history of poor oral hygiene care. The oral hygiene instruction included the use of fluoridated toothpaste supplemented with daily rinse of $0.05 \%$ sodium fluoride mouthrinse. A systematic review found that there was some evidence that the use of daily topical fluoride would reduce occurrence and severity of white spot lesion (12). The paper also recommended the daily rinse of $0.05 \%$ sodium fluoride mouthrinse by patients with fixed appliances. Fortunately the patient had maintained a good oral hygiene status throughout treatment. At debond, new signs of decalcification were not detected.

Treatment was done on an extraction basis to control the antero-posterior displacement (6) and relieve the crowding. Initial treatment involved proclination of the labial segment and expansion of the arch to improve the transverse relationship. The amount of space gained was limited with an estimation of $0.5 \mathrm{~mm}$ to $0.6 \mathrm{~mm}$ of space gained for each millimetre of posterior arch width change and an average of $0.5 \mathrm{~mm}$ to $1 \mathrm{~mm}$ of space gained for a $5^{\circ}$ change of two to four maxillary incisors $(13,14)$ which were not sufficient to relieve the severe upper arch crowding. Furthermore, excessive proclination of the incisors would also have reduced the overbite, thus reducing the stability of the crossbite correction. It may also have further reduced the smile line, thereby affecting the smile aesthetics. As the mid-treatment cephalogram showed that the upper incisors had proclined by $8.7^{\circ}$ to an inclination of $128.2^{\circ}$, any further proclination for space creation may have also been detrimental to the periodontal health of the incisors. A long term follow-up study of Class III patients treated orthodontically, whereby the labial segment was greatly compensated compared with randomly selected Class I and Class II cases, found increased morbidity measured by labial gingival recession and tooth mobility in functional occlusion (15). Although Burns and colleagues (2010) found that camouflage treatment could be done with various tooth movement without deleterious effect on the periodontium, they suggested that the upper and lower incisal limits for dental compensation in Class III cases to be $120^{\circ}$ to the sella-nasion line and $80^{\circ}$ to the mandibular plane, respectively (16).

It has been advocated that the use of labial root torque could advance the 'A' point (17). On hindsight, this could have been done with the use of prescriptions with less torque such as the Andrews $\left(7^{\circ}\right)$ or Roth $\left(12^{\circ}\right)$ or by inverting the brackets to increase the labial root torque i.e. Andrews $\left(-7^{\circ}\right)$, Roth $(-120)$ and MBT $\left(-17^{\circ}\right)$ (18). Nevertheless, uprighting the upper labial segment in cases with skeletal Class III dysplasia needed to be done judiciously particularly when they would be retracted during space closure, as this may have been at the expense of excessive retroclination of the lower labial segment which may not be favourable for the health of the periodontium. The $\mathrm{MBT}^{\mathrm{TM}}$ was the prescription of choice for the pre-adjusted edgewise appliance in this case to balance and prevent excessive compensation in either labial segment. The prescription also offered higher torque value for the upper left lateral incisor bracket $\left(10^{\circ}\right)$ compared with the Andrews $\left(3^{\circ}\right)$ and Roth $\left(8^{\circ}\right)$ when inverted to facilitate more labial root torque movement of the palatally erupted tooth.

In order to camouflage Class III cases, it has been advocated to transpose the lower canine brackets which would effectively facilitate the dentoalveolar movements and reduce the anchorage requirements (18). In this case, the lower canine brackets were not transposed to prevent over compensation of the dentoalveolar relationship. Instead lower canine lacebacks were tied to facilitate antero-posterior control of the dentoalveolar movements during levelling and aligning. In a clinical control trial, canine lacebacks were found to prevent lower labial segment proclination in the order of $2.5 \mathrm{~mm}$ (19). However, it should be noted that the use of lacebacks have been questioned with a randomised control trial finding no difference of canine lacebacks to prevent lower incisor proclination but found a statistically significant difference of more mesial movement of the molars (20). Thus, in order to reduce the amount of mesial movement of the molars, the lower second molars were also included in the canine lacebacks to increase the posterior anchorage. The mechanics of canine lacebacks in this case also had another added advantage of facilitating distal root tipping of the lower canines to bring the roots closer to the lower second premolar roots, thus preventing space from reopening post-treatment.

The working archwire used was upper 0.019 " $\mathrm{x}$ 0.025 " stainless steel archwire. However, in the lower arch, the archwire used was 0.020 " stainless steel. A $0.019 " \mathrm{x} 0.025$ " stainless steel may risk to procline the lower incisors despite the MBT labial root torque $\left(-6^{\circ}\right)$ prescription when the archwire is inserted in the $0.022 " \mathrm{x} 0.028$ " slot of an existing retroclined lower labial segment, which would not have been favourable for the overjet correction. The upper centreline was corrected by the effect of the reciprocal anchorage via the use of nickel titanium push coil spring on a rigid stainless steel archwire which was used to facilitate space opening for the upper left lateral incisor. Spaces were closed using elastomeric powerchains. Powerchains were not only cheaper than nickel titanium coil spring but were as effective for space closure. Although the rate of space closure have been found to be slightly faster with nickel titanium coil spring ( $0.81 \mathrm{~mm}$ per month or $0.26 \mathrm{~mm}$ per week) compared with powerchains $(0.58 \mathrm{~mm}$ per month or $0.21 \mathrm{~mm}$ per week), the differences were not statistically significant $(\mathrm{p}>0.05)(21,22)$. 
Upper arch expansion was initially done with a quad helix, which was removed prior to space closure to reduce the posterior anchorage and encourage mesial movement of the upper buccal segment. Although the upper second permanent molars were in crossbite, they were not initially bonded to monitor the overbite control. Expansion of the upper buccal segment increased the risk for the palatal cusps to drop, thereby reducing the overbite and stability of the crossbite correction. The more posterior the tooth, the more significant this effect would have been in reducing the overbite. Although it may have been prudent to leave the upper second permanent molars in crossbite, it was decided to correct the crossbite due to the presence of a displacement, which prevented proper interdigitation of the occlusion. When the upper second permanent molars were bonded, a light nickel titanium archwire was placed to align the teeth. The jockey arch was inserted into the extra-oral traction tubes of the upper first molar bands to simultaneously support the upper arch and facilitate the crossbite correction during the later part of the treatment. Both the quad helix and the jockey arch were as effective for crossbite correction (23). McNally and colleagues (2005) also reported that patients found both appliances as uncomfortable with the quad helix impinging on the tongue while the jockey arch on the cheeks but more patients disliked the appearance of the jockey arch compared with the quad helix. As anticipated, crossbite correction of the posterior segment had reduced the overbite resulting in lateral open bites, which was addressed by the use of interarch box elastics. On hindsight, this could have also been improved by application of buccal root torque on the molars to lift the palatal cusps and increase the overbite.

Stability of the crossbite correction was favourable provided that there was positive overbite and good posterior intercuspation $(24,25)$. The posterior teeth were still not fully interdigitated at debond. However, this was expected to improve with occlusal settling during the retention period. There was some evidence to show that occlusal settling with Hawley retainers was better than clear overlay retainers after 3 months of full time wear (26), although the study have been criticised to be weak and unrealiable (27). The lower incisors had further retroclined beyond the normal limits to compensate for the underlying skeletal discrepancy. It has been advocated that for stability of treatment the lower incisor position should remained unchanged (28). In view of the significant change in the lower incisor position, fixed bonded retainer was placed as a long term retention strategy $(6,24)$. The retention regime for the Hawley retainers was 6 months full time followed by 6 months night time wear. Recent randomised control trial found that the regimen to wear Hawley retainers full time for 1 year were just as effective with the regimen of 6 months full time and 6 months night time wear (29). Therefore the choice of regimen was based on the clinician's preference. The gingival level was monitored throughout treatment. Fortunately, there was no gingival recession despite the significant change in the lower incisor inclination. This may be due to thickness of the gingival biotype that had favourably not caused any recession. Nevertheless, long term follow up is required to monitor the health of the periodontium for any signs of deterioration (15).

\section{CONCLUSION}

The outcome of the treatment was good and the patient was pleased with the overall aesthetics. The prognosis for stability was expected to be favourable although the periodontal health of the lower labial segment needed long term monitoring. It is important for clinicians to recognise the limitations of treatment and inform the patients of the risks involved and treatment alternatives prior to commencing treatment. Treatment mechanics should be properly employed based on scientific principles and evidences for successful results.

\section{REFERENCES}

1. British Standards Institutes. Glossory of Dental terms (BS4492). BSI London; 1983.

2. Mossey PA. The heritability of malocclusion: part 2. The influence of genetics in malocclusion. $\mathrm{Br} \mathrm{J}$ Orthod 1999;26(3):195-203.

3. Mandall N, DiBiase A, Littlewood S, Nute S, Stivaros N, McDowall R, et al. Is early class III protraction facemask treatment effective? A multicentre, randomized, controlled trial: 15month follow-up. J Orthod 2010;37(3):149-61.

4. Baccetti T, Franchi L, McNamara JA, Jr. Treatment and posttreatment craniofacial changes after rapid maxillary expansion and facemask therapy. Am J Orthod Dentofacial Orthop 2000;118(4):404-13.

5. Westwood PV, McNamara JA, Jr., Baccetti T, Franchi L, Sarver DM. Long-term effects of Class III treatment with rapid maxillary expansion and facemask therapy followed by fixed appliances. Am J Orthod Dentofacial Orthop 2003;123(3):30620.

6. Proffit WR, Fields HW, Sarver DM. Contemporary Orthodontics. Fourth ed: Mosby, Inc; 2007.

7. Kerr WJ, Miller S, Dawber JE. Class III malocclusion: surgery or orthodontics? Br J Orthod 1992;19(1):21-4. 
8. Baccetti N, Franchi L, McNamara JA. The Cervical Vertebral Maturation (CVM) Method for the Assessment of Optimal Treatment Timing in Dentofacial Orthopedics. Seminars in Orthodontics 2005;11:119-29.

9. Malaysian Dental Council. Code of Professional Conduct. 1 ed; 1997.

10. Proffit WR, Turvey TA, Phillips C. The hierarchy of stability and predictability in orthognathic surgery with rigid fixation: an update and extension. Head Face Med 2007;3:21.

11. Artun J, Brobakken BO. Prevalence of carious white spots after orthodontic treatment with multibonded appliances. Eur J Orthod 1986;8(4): 229-34.

12. Benson PE, Parkin N, Millett DT, Dyer F, Vine S, Shah A. Fluorides for the prevention of white spots on teeth during fixed brace treatment. Cochrane Database of Systematic Reviews 2004; Art. No.: CD003809(3):DOI: 10.1002/ 14651858.CD003809.pub2.

13. Kirschen RH, O'Higgins E A, Lee RT. The Royal London Space Planning: an integration of space analysis and treatment planning: Part I: Assessing the space required to meet treatment objectives. Am J Orthod Dentofacial Orthop 2000;118(4):44855.

14. O'Higgins EA, Lee RT. How much space is created from expansion or premolar extraction? J Orthod 2000;27(1):11-3.

15. Sperry TP, Speidel TM, Isaacson RJ, Worms FW. The role of dental compensations in the orthodontic treatment of mandibular prognathism. Angle Orthod 1977;47(4):293-9.

16. Burns NR, Musich DR, Martin C, Razmus T, Gunel E, Ngan P. Class III camouflage treatment: what are the limits? Am J Orthod Dentofacial Orthop 2010;137(1):9 e1-9 e13.

17. Catania JA, Cohen BD, Deeney MR. The use of labial root torque and the tie-forward technique in the treatment of maxillary skeletal retrusion and severe arch length discrepancy. Am J Orthod Dentofacial Orthop 1990;98(1):12-8.
18. Thickett E, Taylor NG, Hodge T. Choosing a preadjusted orthodontic appliance prescription for anterior teeth. J Orthod 2007;34(2):95-100.

19. Robinson SN. An Evaluation of the Changes in Lower Incisor Position During the Initial Stages of Clinical Treatment Using a Preadjusted Edgewise Appliance [MSc Thesis]: University of London; 1989.

20. Irvine R, Power S, McDonald F. The effectiveness of laceback ligatures: a randomized controlled clinical trial. J Orthod 2004;31(4):303-11.

21. Nightingale C, Jones SP. A clinical investigation of force delivery systems for orthodontic space closure. J Orthod 2003;30(3):229-36.

22. Dixon V, Read MJ, O'Brien KD, Worthington HV, Mandall NA. A randomized clinical trial to compare three methods of orthodontic space closure. J Orthod 2002;29(1):31-6.

23. McNally MR, Spary DJ, Rock WP. A randomized controlled trial comparing the quadhelix and the expansion arch for the correction of crossbite. J Orthod 2005;32(1):29-35.

24. Johnston C, Burden D, Morris D. Clinical Guidelines: Orthodontic Retention. The Royal College of Surgeons of England 1999 (2008 Revision):1-9.

25. Kaplan H. The logic of modern retention procedures. Am J Orthod Dentofacial Orthop 1988;93(4):325-40.

26. Sauget E, Covell DA, Jr., Boero RP, Lieber WS. Comparison of occlusal contacts with use of Hawley and clear overlay retainers. Angle Orthod 1997;67(3):223-30.

27. Littlewood SJ, Millett DT, Doubleday B, Bearn DR, Worthington HV. Orthodontic retention: a systematic review. J Orthod 2006;33(3):205-12.

28. Mills JR. The stability of the lower labial segment. A cephalometric survey. Dent Pract Dent Rec 1968;18(8):293-306.

29. Shawesh M, Bhatti B, Usmani T, Mandall N. Hawley retainers full- or part-time? A randomized clinical trial. Eur J Orthod 2010;32(2):165-70. 